\title{
ARTICLE \\ Profound alteration in reward processing due to a human polymorphism in CHRNA5: a role in alcohol dependence and feeding behavior
}

\author{
Morgane Besson ${ }^{1}$, Benoît Forget ${ }^{1,2}$, Caroline Correia ${ }^{1,3}$, Rodolphe Blanco ${ }^{1}$ and Uwe Maskos ${ }^{1}$
}

\begin{abstract}
Human genetic variation in the nicotinic receptor gene cluster CHRNA5/A3/B4, in particular the non-synonymous and frequent CHRNA5 variant rs16969968 (a5SNP), has an important consequence on smoking behavior in humans. A number of genetic association studies have additionally implicated the CHRNA5 gene in addictions to other drugs, and also body mass index (BMI). Here, we model the a5SNP, in a transgenic rat line, and establish its role in alcohol dependence, and feeding behavior. Rats expressing the a5SNP consume more alcohol, and exhibit increased relapse to alcohol seeking after abstinence. This high-relapsing phenotype is reflected in altered activity in the insula, linked to interoception, as established using c-Fos immunostaining. Similarly, relapse to food seeking is increased in the transgenic group, while a nicotine treatment reduces relapse in both transgenic and control rats. These findings point to a general role of this human polymorphism in reward processing, and multiple addictions other than smoking. This could pave the way for the use of medication targeting the nicotinic receptor in the treatment of alcohol use and eating disorders, and comorbid conditions in smokers.
\end{abstract}

Neuropsychopharmacology (2019) 44:1906-1916; https://doi.org/10.1038/s41386-019-0462-0

\section{INTRODUCTION}

Addiction is a psychiatric disorder defined by a loss of control over drug taking and seeking, characterized by chronic relapsing following attempts to quit [1]. Therapies and treatments to alleviate withdrawal symptoms can assist patients in maintaining abstinence, yet there is a critical need for more effective medication addressing relapse prevention [1]. Tobacco and alcohol addictions are the two leading causes of premature death among all main causes of excess mortality [2]. They show strikingly high rates of comorbidity and individuals with these two addictions represent the largest group of polysubstance abusers, although there is a strong lack in both the understanding of the biological basis and therapeutic approaches of such comorbidity $[3,4]$. Nicotine, the main psychoactive substance of tobacco smoke responsible for its addictive properties, acts on the nicotinic acetylcholine receptors (nAChRs). They are pentameric ligand-gated ion channels widely expressed in the brain, where they are composed of a (a2-a6, a7, a9, a10) and $\beta(\beta 2-\beta 4)$ subunits that co-assemble according to various combinations exhibiting distinct brain localizations and functional properties [5]. Over the past 10 years, a plethora of human genetic studies, including Genome-Wide Association Studies (GWAS), have identified a consistent association between a single nucleotide polymorphism (rs16969968) of the CHRNA5 gene encoding the a5 nAChR subunit (a5SNP) and the risk for higher scores for nicotine dependence [6-10]. The a5SNP, very frequent in the general population, is non-synonymous, changing an aspartic acid into an asparagine, and doubles the risk to develop heavy smoking in homozygous carriers [11-13]. We have recently created transgenic rats constitutively expressing the a5SNP, and notably identified increased relapse to nicotine seeking after abstinence in these rats using self-administration (SA) procedures [14]. Because of the strong co-occurrence of tobacco and alcohol dependence, several human candidate gene studies have attempted to identify possible links between the a5SNP and alcohol abuse but with discordant results [15-18]. Such incongruity in human studies can be attributed to several factors, such as varying linkage disequilibrium, population heterogeneity, cohort design, including patients with multiple substance addiction and criteria for phenotyping.

Here, we investigated the impact of the a5SNP on multiple behaviors related to alcohol abuse using drug-naive transgenic rats in complementary preclinical models of alcohol addiction. We notably assessed reinstatement of alcohol seeking after extinction of alcohol SA, a model of relapse with strong translational value [19], in combination with c-Fos immunostaining to correlate neuronal responses to relapse intensity. We also verified whether the addiction-like phenotypes observed in a5SNP rats could be due to impaired alcohol metabolism, locomotor activity or anxiety.

Drug addiction has been proposed to partially result from maladaptive motivation and reward processing, associated with dysfunctions in mechanisms important for the pursuit of natural reinforcers [1]. nAChRs are key players in reward-related mechanisms $[20,21]$, and in vitro studies have shown that the a5SNP causes a partial loss of $n A C h R$ function $[7,22,23]$, notably within the reward pathway [24]. We further hypothesized that the a5SNP

\footnotetext{
${ }^{1}$ Department of Neuroscience, Unité de Neurobiologie Intégrative des Systèmes Cholinergiques, CNRS UMR 3571, Institut Pasteur, 25 rue du Dr Roux, 75015 Paris, France ${ }^{2}$ Present address: Sorbonne Universités, UPMC Université Paris 06, INSERM, CNRS, Neuroscience Paris Seine - Institut de Biologie Paris Seine (NPS - IBPS), 75005 Paris, France ${ }^{3}$ Present address: Laboratoire de Neurosciences Cognitives et Adaptatives, CNRS UMR 7364, Université de Strasbourg, 67000 Strasbourg, France Correspondence: Morgane Besson (morgane.besson@pasteur.fr) or Uwe Maskos (uwe.maskos@pasteur.fr)
} 
may impact reward-related mechanisms not only in the context of drug intake but also in physiological conditions, i.e. during natural reward processing. Interestingly, the a5SNP has been associated with higher body mass index (BMI) in never smokers, but lower $\mathrm{BMI}$ in current smokers, suggesting that this variant may cause alterations in food responding that could be counteracted by smoking [25]. Thus, we also examined the consequences of the a5SNP on appetence and motivation for food in a SA procedure, and assessed the effects of nicotine exposure on food seeking relapse behavior.

\section{MATERIALS AND METHODS}

Animals

Adult male wild-type (WT) rats and rats constitutively carrying the rs16969968 SNP (a5SNP rats) [14], on a Long-Evans background, were used. All experimental procedures were approved by the institutional Animal Care Committee (agreements $\mathrm{N}^{\circ} 0355.02$ and 180021). All efforts were made to minimize animal suffering, and to reduce the number of animals. Details are given in Supplementary Methods.

Intermittent ethanol two-bottle choice paradigm

Drug-naïve rats were given one bottle with $20 \%$ ethanol (EtOH) v/v (Sigma Aldrich, Saint Quentin Fallavier, France) and one bottle with tap water in their home cages according to a weekly intermittent schedule. The acquisition was followed by a quinine adulteration phase (quinine hydrochloride, Sigma-Aldrich, Saint Quentin Fallavier, France) to test aversion-resistant alcohol intake (adapted from [26]). A subgroup of animals was then submitted to four months of withdrawal and re-exposed to $\mathrm{EtOH}$ for two choice sessions. Details are given in Supplementary Methods.

\section{Ethanol operant oral self-administration procedure}

Drug-naïve rats were submitted to operant oral SA of $12 \% \mathrm{EtOH}$ v/v (Fisher Scientific, Ilkirch, France) in chambers equipped with two levers (Med Associates, St. Albans, Vt., USA). Habituation: Rats were exposed to progressively increased concentrations of $\mathrm{EtOH}$ for habituation. Acquisition: Rats acquired EtOH SA under fixed ratio (FR) schedules of reinforcement from FR1 to FR5. The unit dose was a $0.1 \mathrm{~mL}$ drop of $12 \% \mathrm{EtOH}$, associated with a $10 \mathrm{~s}$ presentation of a visual cue (light) above the active lever (AL). Progressive Ratio responding: Rats were switched to a progressive ratio (PR) schedule of reinforcement during three consecutive sessions wherein the response requirement increased with each successive EtOH reinforcement. Dose-response curve: Rats were switched back to FR5 for a few days before being tested for SA of different doses of EtOH $(6,12,18$, and 30\%). Extinction: Rats were then submitted to an extinction phase where responses on levers were recorded, but did not result in $\mathrm{EtOH}$ or visual cue delivery. Reinstatement of EtOH seeking: A cue-induced reinstatement test was conducted. Rats were submitted to another extinction, and tested for "EtOH + cue"-induced reinstatement of EtOH seeking. Rats were then submitted to a last extinction, and a part of them, was tested again for "EtOH + cue"-induced reinstatement of $\mathrm{EtOH}$ seeking while the other part was submitted to an extinction session. Details are given in Supplementary Methods.

Immunofluorescence and c-Fos counting Following the last "EtOH + cue"-induced reinstatement of EtOH seeking or extinction sessions, brains were extracted and processed for c-Fos immunofluorescence and quantification. Details are given in Supplementary Methods.

Blood ethanol concentration measurements

Drug naïve rats received an intraperitoneal injection of $\mathrm{EtOH}$ $(2 \mathrm{~g} / \mathrm{kg})$ and were sacrificed at different time points post-injection
$(15,30,90$, and $180 \mathrm{~min})$ just before blood collection. Serum was assayed for EtOH content using an Ethanol Assay Kit (MAKO76, Sigma-Aldrich, Saint Quentin Fallavier, France). Details are given in Supplementary Methods.

Locomotor activity and anxiety-like behavior measurement Locomotor activity and anxiety-like behavior of rats were recorded for $30 \mathrm{~min}$ in a square open-field. Anxiety-like behavior was further evaluated for $5 \mathrm{~min}$ in the dark-light box (DLB) test in the same drug-naïve rats. Details are given in Supplementary Methods.

Food operant self-administration procedure

Drug-naïve rats were submitted to operant SA of food $(45 \mathrm{mg}$ pellets, rodent purified diet F0021, Bio-Serv, Morangis, France) in chambers similar to those used for EtOH SA. Acquisition: Rats acquired food SA under FR schedules of reinforcement and the unit dose was one food pellet delivered into a magazine between the two levers associated with a $10 \mathrm{~s}$ presentation of a visual cue (light) above the AL. Progressive Ratio responding: Rats were switched to a PR schedule of reinforcement during three consecutive sessions wherein the response requirement increased with each successive reinforcement. Extinction: Rats were switched back to FR5 for a few days before being submitted to an extinction phase where responses on the levers were recorded, but did not result in food or visual cue delivery. Reinstatement of food seeking: rats were submitted to a food-induced reinstatement test, and to another extinction, before being tested for cue-induced reinstatement. Rats were then submitted to a last extinction phase before being tested again for food-induced reinstatement. For this last reinstatement session, half of the rats received a sub-cutaneous injection of nicotine ((-)Nicotine hydrogen tartrate, Sigma-Aldrich, St Louis, Mo., USA) dissolved in $\mathrm{NaCl} 0.9 \%$, at the dose of $0.1 \mathrm{mg} /$ $\mathrm{kg}$ (free base), while the other half received only $\mathrm{NaCl} 0.9 \%, 5 \mathrm{~min}$ before starting the session. Details are given in Supplementary Methods.

\section{Statistics}

All data were analyzed with Statistica (StatSoft, Inc., France). For two-group comparisons, data were analyzed with unpaired Student's $t$ or Mann-Whitney U tests when normality and variance homogeneity conditions were not met for parametric test use. Two- and three-way repeated measures ANOVAs were used (results reported in Supplementary Tables 1-5). Significant main effects $(p<0.05)$ were further analyzed using Bonferroni for multiple comparisons post hoc tests. Details are given in Supplementary Methods.

\section{RESULTS}

Ethanol intake and preference in an intermittent two-bottle choice procedure in a5SNP and WT rats

To examine the impact of the a5SNP on voluntary alcohol consumption, we first submitted rats carrying the a5SNP and WT rats to an intermittent two-bottle choice paradigm with limited access to EtOH (Fig. 1a). On the first day, EtOH preference was higher in a5SNP rats with a similar trend observed for EtOH intake (Fig. S1a). EtOH preference and intake progressively increased, reaching a plateau of $\pm 60 \%$ for EtOH preference (Fig. $1 \mathrm{~b}$ ). Global $\mathrm{EtOH}$ preference and intake were not different between genotypes (see Table S1 for all ANOVA results). Yet, there was a significant groupXsession interaction for both parameters, indicating different patterns of $\mathrm{EtOH}$ consumption over time between groups. No evolution over time nor group differences were observed on the preference for the $\mathrm{EtOH}$ side on days where only water was available (Fig. S1b). Rats were then submitted to several sessions of adulterated $\mathrm{EtOH}$ consumption induced by concomitant exposure to quinine, a procedure proposed to model pathological consumption of alcohol [26] (Fig. 1c, d). Quinine 
a

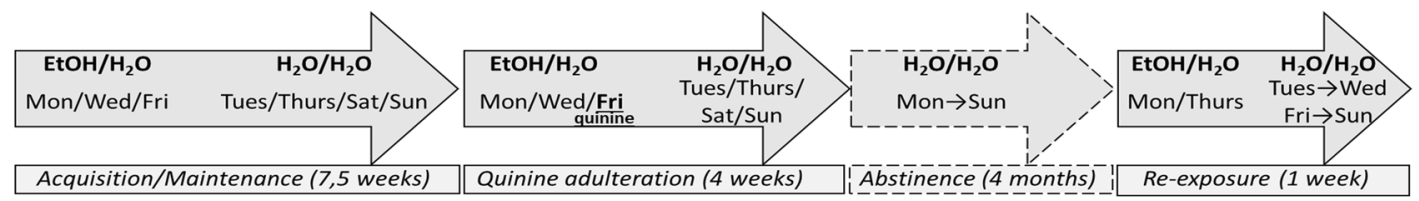

b
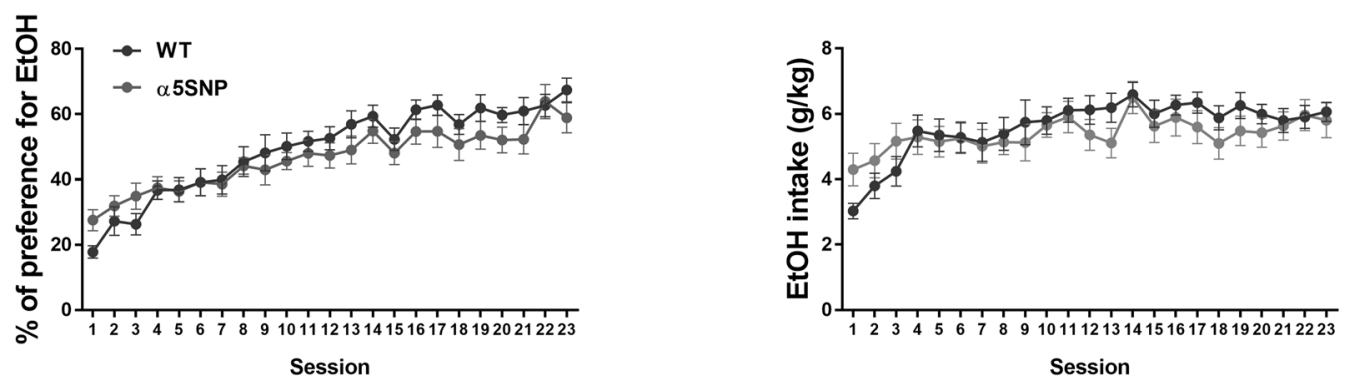

C

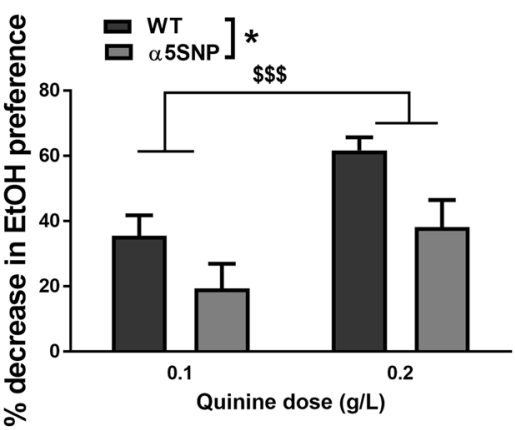

d
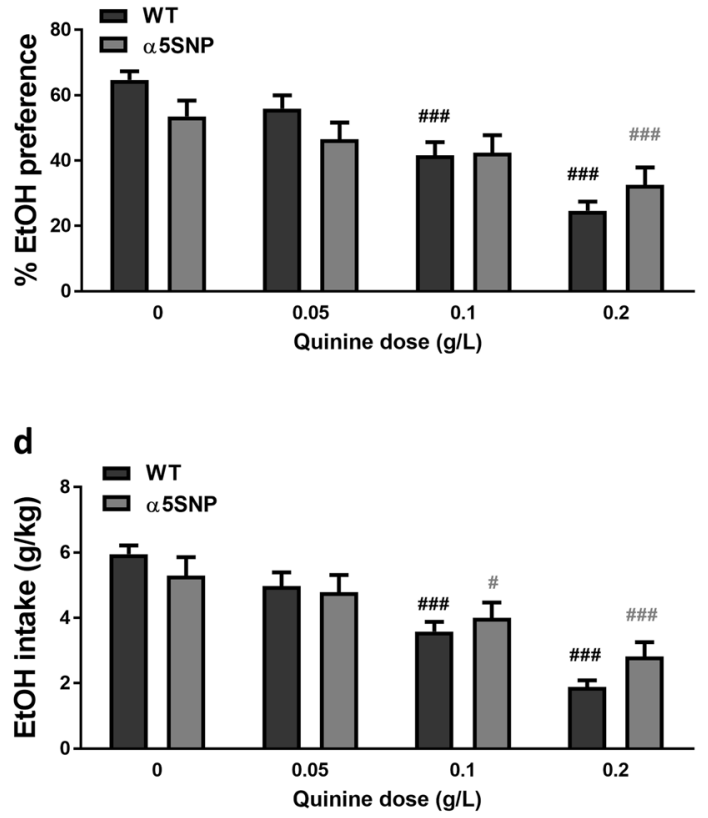

e

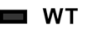

$\square \alpha 5$ SNP

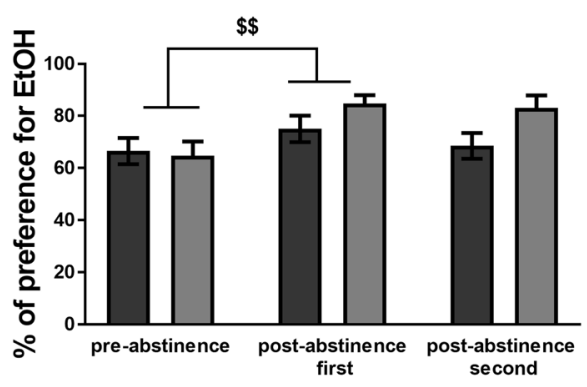

dose-dependently decreased EtOH preference and intake. No global differences were obtained between genotypes, but a5SNP and WT rats differentially adapted their EtOH consumption according to increasing quinine doses. Post hoc tests showed that $\mathrm{EtOH}$ preference was decreased from the medium dose in
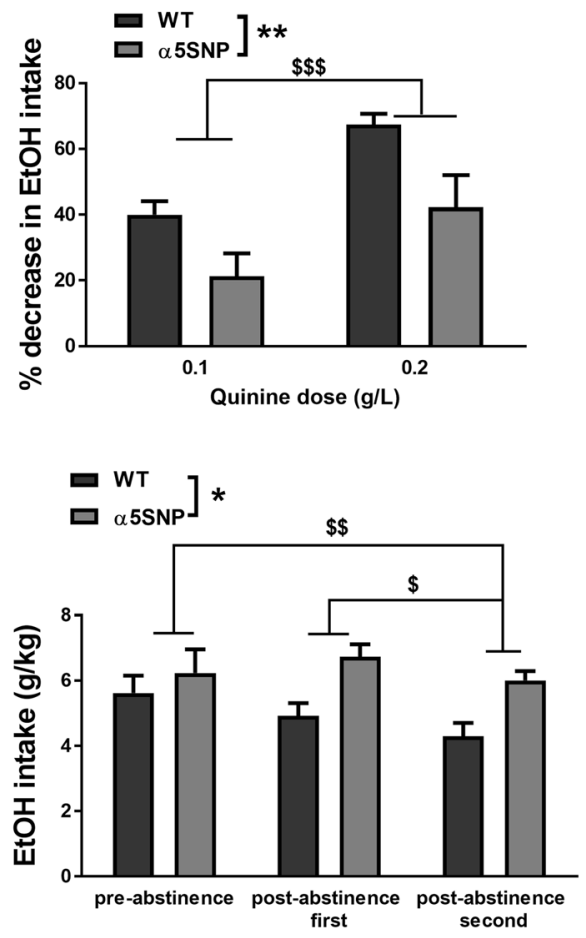

WTs while this was only the case at the highest dose of quinine in a5SNP rats (Fig. 1c, left). The percentage of decrease in EtOH preference was higher at $0.2 \mathrm{~g} / \mathrm{L}$ compared to $0.1 \mathrm{~g} / \mathrm{L}$, but lower in a5SNP rats compared to WTs (Fig. 1c, right). EtOH intake was decreased from the medium dose in both groups (Fig. 1d, left). Yet 
Fig. 1 Ethanol intake and preference in a two-bottle choice procedure. a Scheme of the procedure. $\mathbf{b}$ Percentage of EtOH preference (left) and total intake (right) during acquisition in WT $(n=15)$ and $\alpha 5$ SNP $(n=14)$ rats [two-way repeated measure ANOVAs]. c Percentage of EtOH preference (left) [two-way repeated measure ANOVAs and Bonferroni post hoc. WTs: 0 vs. $0.1 \mathrm{~g} / \mathrm{L}: p<0.0001,0 \mathrm{vs.} 0.2 \mathrm{~g} / \mathrm{L}: p<0.0001 ; \alpha 5 \mathrm{SNPs}: 0$ vs. $0.2 \mathrm{~g} / \mathrm{L}: p<0.001$ ] and percentage of decrease in $\mathrm{EtOH}$ preference (right) [two-way repeated measure ANOVAs] during quinine adulteration in WT $(n=15)$ and $\alpha 5$ SNP $(n=13)$ rats. $\mathbf{d}$ Total EtOH intake (left) [two-way repeated measure ANOVAs and Bonferroni post hoc. WTs: 0 vs. 0.1 $\mathrm{g} / \mathrm{L}: p<0.0001,0$ vs. $0.2 \mathrm{~g} / \mathrm{L}: p<0.0001 ; \alpha 5 S N P s: 0$ vs. $0.1 \mathrm{~g} / \mathrm{L}: p<0.05,0$ vs. $0.2 \mathrm{~g} / \mathrm{L}: p<0.0001]$ and percentage of decrease in EtOH intake (right) [two-way repeated measure ANOVAs] during quinine adulteration in WT $(n=15)$ and $\alpha 5 S N P(n=13)$ rats. e Percentage of EtOH preference (left) [two-way repeated measure ANOVAs and Bonferroni post hoc. pre- vs. 1st post-abstinence: $p<0.01]$ and total intake (right) [two-way repeated measure ANOVAs and Bonferroni post hoc. pre- vs. 2nd post-abstinence: $p<0.01$, 1 st post- vs. 2nd post-abstinence: $p<$ 0.05 ] during pre-, 1 st and 2nd post-abstinence re-exposure sessions in WT $(n=8)$ and $\alpha 5 S N P(n=6)$ rats. Data are mean + s.e.m. Group effect (WT vs. $\alpha 5$ SNP): ${ }^{*} p<0.05,{ }^{* *} p<0.01$. Session effect (quinine vs. no quinine for each dose): ${ }^{*} p<0.05,{ }^{\# \#} p<0.001$ in WT (black) or $\alpha 5 S N P$ rats (gray). Quinine dose or session effect (pre- vs. post-abstinence, or post-abstinence 1st vs. 2nd) in both groups: ${ }^{\$} p<0.05$, ${ }^{\$ \$} p<0.01,{ }^{\$ \$} p<0.001$

the percentage of decrease in $\mathrm{EtOH}$ intake, higher at $0.2 \mathrm{~g} / \mathrm{L}$ compared to $0.1 \mathrm{~g} / \mathrm{L}$, was lower in a5SNP rats compared to WTs (Fig. 1d, right). A much stronger effect of quinine was observed on the preference for the EtOH side on days where only water was available, with no differences between groups, suggesting that the resistance to quinine adulteration observed in a5SNPs was EtOH-specific (Fig. S1c). Rats then underwent 4 months of abstinence before being re-exposed to EtOH during two choice sessions. Preference for EtOH was higher during the first reexposure as compared to the last session before withdrawal in both groups (Fig. 1e, left). Such difference was not observed for $\mathrm{EtOH}$ intake (Fig. 1e, right), because of a parallel increase in animals' weight (Fig. S1d). Finally, EtOH intake was lower during the second re-exposure session compared to both pre- and first post-abstinence sessions, and a5SNP rats consumed more EtOH than WTs overall (Fig. 1e, right).

Ethanol operant self-administration in a5SNP and WT rats To further characterize the impact of the a5SNP on EtOH addictionlike behaviors, we next submitted drug-naïve a5SNP and WT rats to a chronic EtOH oral SA procedure in operant chambers, which examines multiple aspects of EtOH abuse with good face validity [27] (Fig. 2a). Both groups acquired EtOH SA with a progressive increase in AL presses to obtain a drop of a $12 \% \mathrm{EtOH}$ solution $(0.1$ $\mathrm{mL}$ ) (see Table S2 for all ANOVA results). However, a5SNP rats selfadministered more EtOH than WTs, with increased number of lever presses (Fig. 2b, left), and higher EtOH intake (Fig. 2b, right). When tested under a PR schedule of reinforcement to further measure EtOH motivational effects, a5SNP rats reached a higher break point than WTs (Fig. 2c). Rats were then tested for SA of multiple doses of EtOH. a5SNP and WT rats both increased but differentially adapted their EtOH intake with an increase in dose (Fig. 2d). Post hoc tests showed that EtOH intake was still increased from the next-to-last to the last doses in a5SNP rats while it was stabilized in WTs. An extinction was then conducted where $A L$ presses no longer resulted in $\mathrm{EtOH}$ or visual cue delivery, inducing a progressive decrease of $A L$ responding in both groups, with the global number of lever presses remaining higher in a5SNP rats as compared to WTs (Fig. 2e). After stabilization of lever responding, rats were submitted to several sessions of reinstatement of EtOH seeking. WTs and a5SNPs exhibited a similar cue-induced reinstatement of $\mathrm{EtOH}$ seeking as observed on AL presses (Fig. 2f, left), and on relapse index (calculated as the subtraction of the number of $\mathrm{AL}$ presses averaged for the three last extinction sessions from the number of $A L$ presses during the relapse session) (Fig. 2f, right). In contrast, a5SNPs had a significantly higher level of reinstatement than WTs when re-exposed to EtOH additionally to the cue, as observed on both $\mathrm{AL}$ presses (Fig. $2 \mathrm{~g}$, left) and relapse index (Fig. 2g, right). Post hoc confirmed increased number of AL presses in both groups after $\mathrm{EtOH}+$ cue re-exposure and higher $\mathrm{AL}$ responding in a5SNP rats compared to WTs in relapse conditions. These data confirm that the rs16969968 enhances addiction-like behaviors for $\mathrm{EtOH}$, and particularly the intensity of relapse to $\mathrm{EtOH}$ seeking after extinction, similarly to what we previously observed for nicotine [14].
Neuronal activation associated with EtOH + cue-induced reinstatement of EtOH seeking in a5SNP and WT rats

To identify the brain structures implicated in the impact of the rs16969968 on relapse to EtOH seeking, we examined the expression of the c-Fos immediate early gene product, a marker of neuronal activation [28], associated with $\mathrm{EtOH}+$ cue-induced reinstatement of EtOH seeking in multiple areas. Rats underwent a new phase of extinction before being submitted to either a session of relapse or a session of extinction for subsequent c-Fos quantification. We confirmed higher level of $\mathrm{EtOH}+$ cue-induced reinstatement in a5SNP rats compared to WTs (Fig. 3a) (see Table S3 for all ANOVA results). In rats submitted to an extinction session, AL responding was still different between groups, but there was no session effect (Fig. 3b). EtOH + cue-induced reinstatement of EtOH seeking was associated with an increase of c-Fos expression in several areas in both groups compared to extinction, namely the cingulate cortex, area $2(\mathrm{Cg} 2)$, the prelimbic cortex (PrL), the nucleus accumbens core (NAcbC) and shell (NAcbS), the paraventricular thalamus (PVTh), and the lateral hypothalamus (LH) (Fig. 3c). Moreover, a strong increase in c-Fos expression was observed in the anterior agranular insula (Al) of a5SNP rats during EtOH seeking relapse compared to extinction, while this area was not activated in relapsing WT rats (Fig. 3c, d). Furthermore, the number of c-Fos-positive cells in the Al was correlated with the level of reinstatement of $\mathrm{EtOH}$ seeking (Fig. 3e).

EtOH metabolism, locomotor activity and anxiety-like behaviors in a5SNP and WT rats

Since possible direct interactions between $\mathrm{EtOH}$ and nACRs have been reported [29,30], we verified in drug-naïve animals that the $\mathrm{EtOH}$ addiction-like phenotypes observed in a5SNP rats were not due to differences in $\mathrm{EtOH}$ metabolism. Blood EtOH concentrations were similar between groups at all time-points following a $2 \mathrm{~g} / \mathrm{kg} \mathrm{EtOH}$ injection (Fig. 4a) (see Table S4 for ANOVA results). High response to novelty and trait-anxiety have been shown to confer vulnerability to drug SA and addiction, including to alcohol abuse where alcohol may be used as a form of emotional selfmedication [31]. Thus we next examined whether locomotor reactivity to novelty and anxiety-like behaviors were altered in drug naïve a5SNP rats. The open-field distance travelled and velocity, reflecting locomotor activity, and \% of time spent in the center, more related to anxiety-like behavior, were similar between genotypes (Fig. 4b). Anxiety-like behaviors in the DLB were not altered in a5SNP rats as suggested by similar percentage of time spent in the light side, number of transitions and latency to first entry into the light side (Fig. 4c). Thus, the $\mathrm{EtOH}$ addictionlike profile observed in a5SNP rats does not seem due to an impact of the rs 16969968 on EtOH metabolism or on predisposing behavioral endophenotypes.

Operant self-administration of food in a5SNP and WT rats Our data indicate that the rs16969968 impacts addiction-like processes not only for nicotine, as previously observed [14], but also for alcohol. We therefore wanted to establish whether this 
a

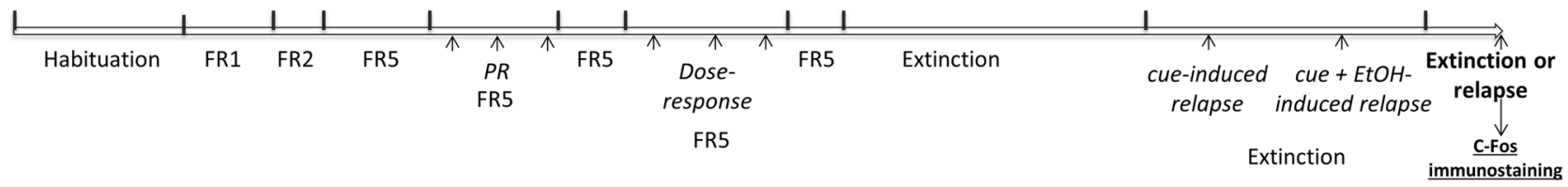

b

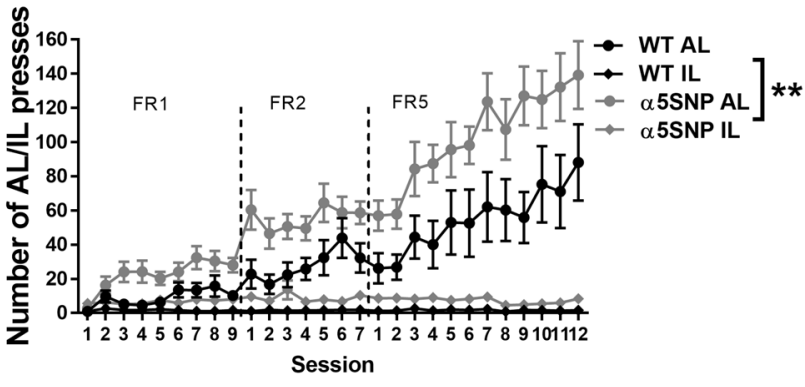

C

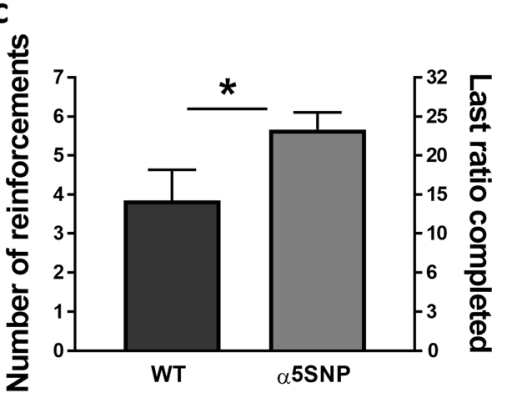

f

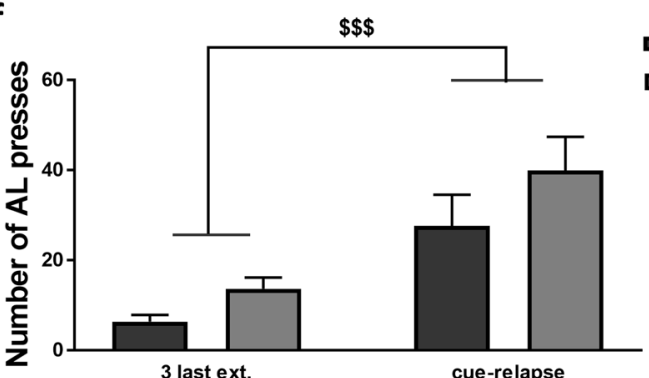

g

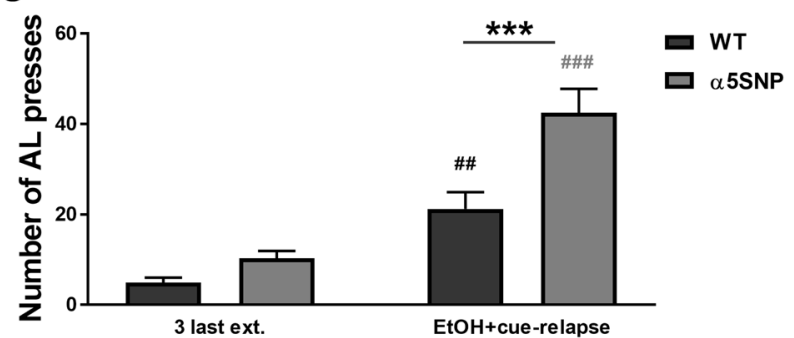

WT

口 $\alpha 5 \mathrm{SNP}$

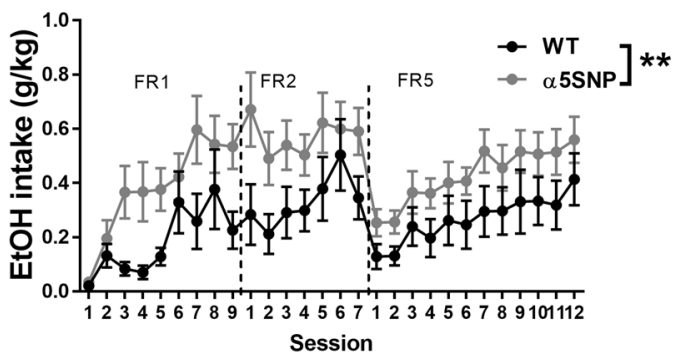

e

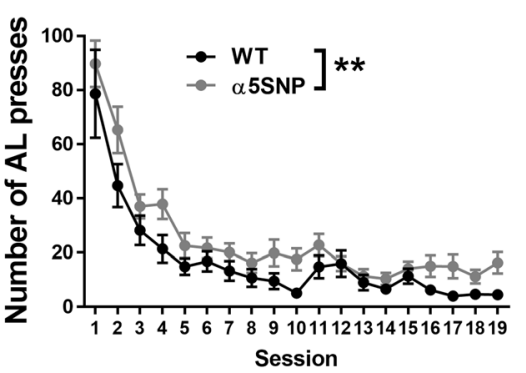

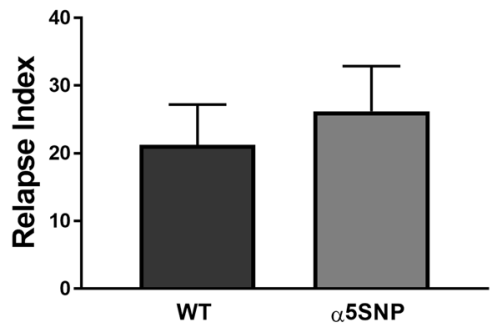

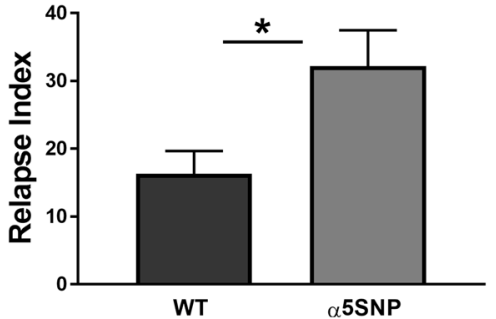

Fig. 2 Ethanol (EtOH) operant self-administration. a Scheme of the procedure. b Number of active (AL) and inactive (IL) lever presses (left) [three-way repeated measure ANOVAs] and total EtOH intake (right) [two-way repeated measure ANOVAs] during acquisition in WT ( $n=15$ ) and $\alpha 5$ SNP $(n=16)$ rats. c Number of reinforcements and last ratio completed in WT $(n=15)$ and $\alpha 5$ SNP $(n=16)$ rats under progressive ratio [Mann-Whitney $z=-2.095, p<0.05]$. d Dose-response curve of the amount of EtOH consumed under FR5 schedule in WT $(n=15)$ and $\alpha 5 S N P$ $(n=16)$ rats [two-way repeated measure ANOVAs and Bonferroni post hoc $\alpha 5$ SNPs: $18 \%$ vs. $30 \%, p<0.01$ ]. e Number of AL presses during extinction in WT $(n=15)$ and $\alpha 5 \mathrm{SNP}(n=16)$ rats [two-way repeated measure ANOVAs]. $\mathbf{f}$ Number of AL presses (left) [two-way repeated measure ANOVAs] and relapse index (right) [Unpaired Student's $t$-test. $t_{28}=0.555$, NS] in WT $(n=15)$ and $\alpha 5 S N P(n=15)$ rats during cueinduced EtOH seeking reinstatement. g Number of AL presses (left) [two-way repeated measure ANOVAs and Bonferroni post hoc, WT: ext. vs. rel., $p<0.001 ; \alpha 5$ SNPs: ext. vs. rel., $p<0.0001$; Relapse: WT vs. $\alpha 5$ SNP, $p<0.001$ ] and relapse index (right) [Unpaired Student's $t$-test. $t_{25}=$ $-2.566, p<0.05]$ in WT $(n=14)$ and $\alpha 5 \mathrm{SNP}(n=13)$ rats during EtOH + cue-induced EtOH seeking reinstatement. Data are mean + s.e.m. Group effect (WT vs. $\alpha 5$ SNP): ${ }^{*} p<0.05,{ }^{* *} p<0.01,{ }^{* * *} p<0.001$. Session (ext. vs. rel.) or dose effect: ${ }^{\$ \$ \$} p<0.001$ in both groups, ${ }^{\# \#} p<0.01,{ }^{\# \# \#} p<$ 0.001 in WT (black) or $\alpha 5$ SNP rats (gray) 
a

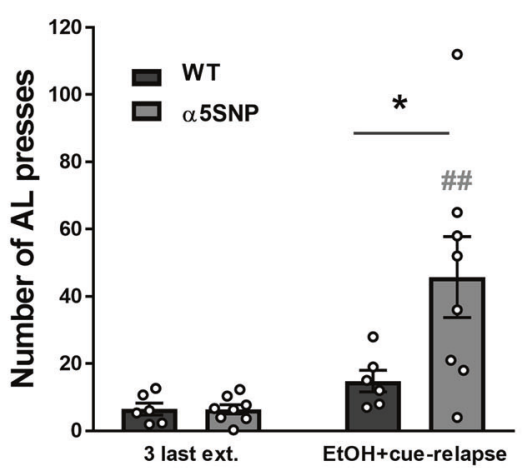

C

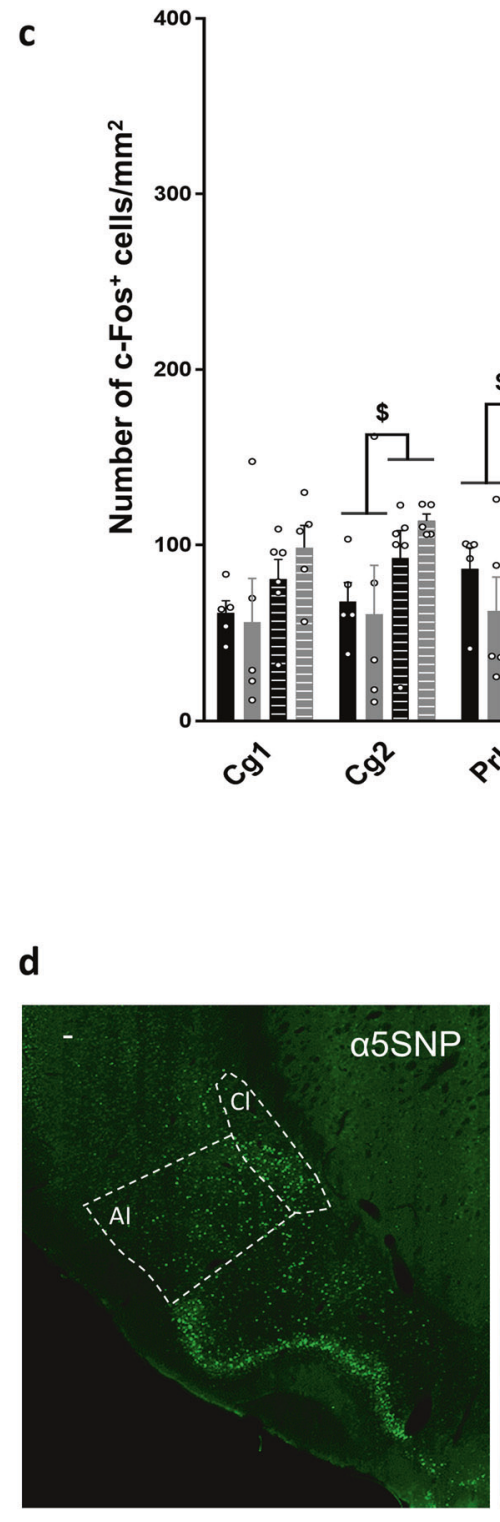

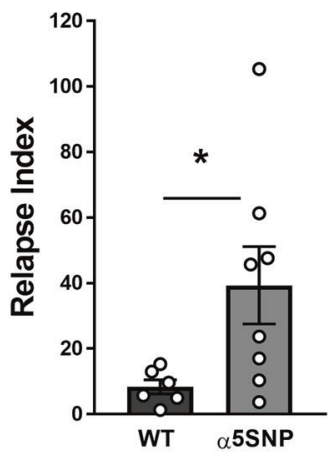

b

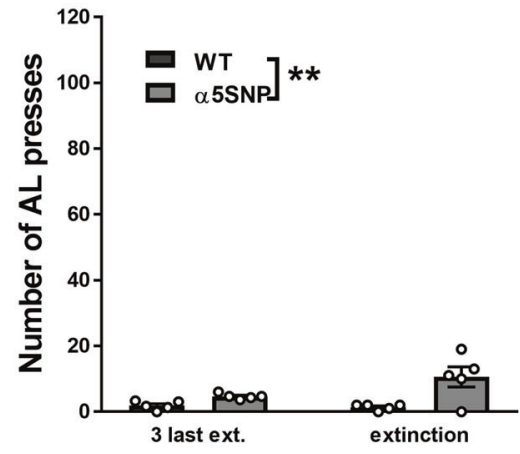

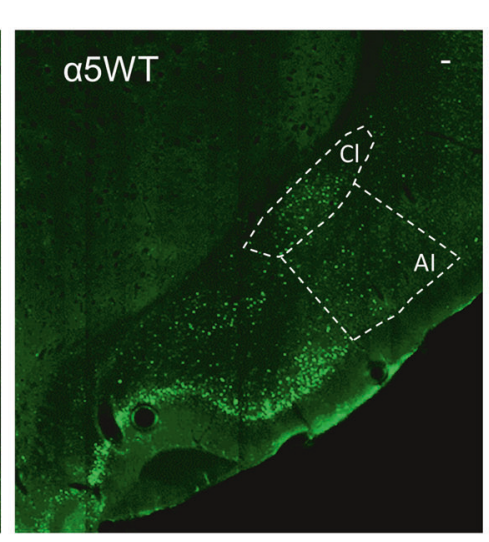

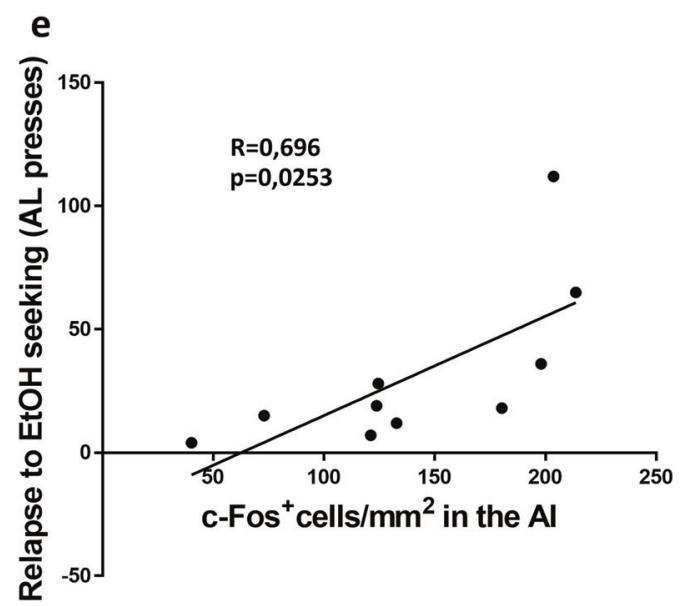

variant may alter reward processing in general and examined if the increased appetitive behavior observed for both nicotine and $\mathrm{EtOH}$ in a5SNP rats may be extended to natural reward. Drugnaïve rats were submitted to a chronic food SA procedure in operant chambers (Fig. 5a). Both groups similarly acquired food SA (Fig. 5b) (see Table S5 for all ANOVA results). Rats were next tested under a PR schedule of reinforcement as another measurement of food reinforcing efficacy. a5SNP rats showed a trend towards higher break point (Fig. 5c). Food SA behavior was then extinguished during a phase where AL presses no longer resulted in food or visual cue delivery, inducing a progressive decrease of AL responding in both groups. However, the number of AL presses was globally higher in a5SNP rats as compared to WTs suggesting a resistance to extinction in a5SNP rats (Fig. $5 \mathrm{~d}$ ). 
Fig. 3 Neuronal activation associated with Ethanol (EtOH) + cue-induced reinstatement of EtOH. a Number of active lever (AL) presses (left) [two-way repeated measure ANOVAs and Bonferroni post hoc $\alpha 5$ SNPs: ext. vs. rel., $p<0.01$; Relapse: WT vs. $\alpha 5 S N P, p<0.05$ ] and relapse index (right) [Mann-Whitney $z=-2.130, p<0.05$ ] in WT $(n=6)$ and $\alpha 5 \mathrm{SNP}(n=8)$ rats during EtOH + cue-induced EtOH seeking reinstatement in rats used for c-Fos quantification. b Number of AL presses in WT $(n=5)$ and $\alpha 5 \mathrm{SNP}(n=5)$ rats during extinction in rats used for c-Fos quantification [two-way repeated measure ANOVAs]. c Levels of expression of c-Fos during EtOH + cue-induced reinstatement of EtOH seeking or extinction in WT $[n=4-6$ (rel.) and 4-5 (ext.)] and $\alpha 5$ SNP [ $n=4-6$ (rel.) and 4-5 (ext.)] rats [two-way repeated measure ANOVAs, and Bonferroni post hoc for Ant. Insula. $\alpha 5$ SNPs: ext. vs. rel., $p<0.0001$; Relapse: WT vs. $\alpha 5 S N P$, $p<0.001]$. d Representative c-Fos immunofluorescence $(\mathrm{X} 20)$ in the anterior insula (AI) and Claustrum (CI) performed on brain slices after reinstatement of EtOH seeking. White bar represents $50 \mu \mathrm{m}$. e Correlation between the number of c-Fos-positive cells in the Al and the level of reinstatement [Two-tailed Spearman $R=0.696 ; p<0.05]$, WT rats $(n=5)$ and $\alpha 5 S N P(n=4)$ are shown. Data are mean + s.e.m. Group effect (WT vs. $\alpha 5 S N P):{ }^{*} p<0.05$,

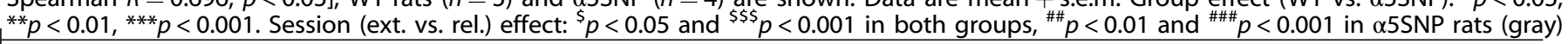

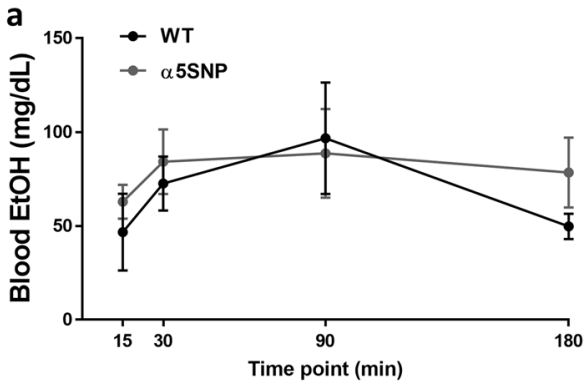

b
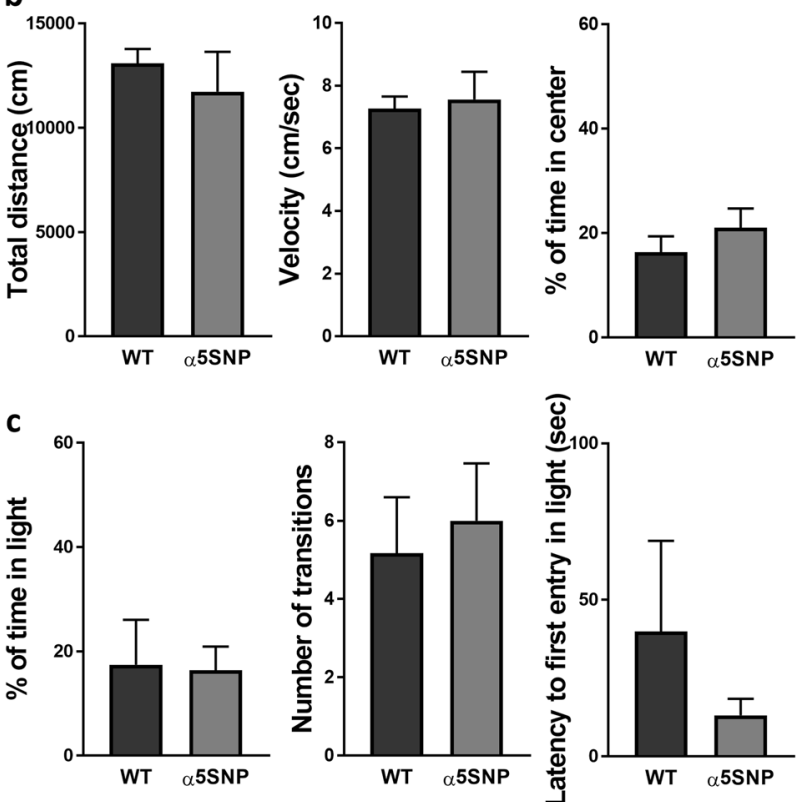

Fig. 4 Ethanol (EtOH) metabolism, locomotor activity and anxietylike behavior. a Blood EtOH concentrations in WT and $\alpha 5$ SNP rats, at 15 (WT $n=4, \alpha 5$ SNP $n=4), 30$ (WT $n=5, \alpha 5 S N P n=5$ ), 90 (WT $n=$ 3, $\alpha 5$ SNP $n=4)$ or $180($ WT $n=5, \alpha 5$ SNP $n=4)$ minutes following intraperitoneal EtOH injection $(2 \mathrm{~g} / \mathrm{kg}$ ) [two-way repeated measure ANOVAs]. b Total distance moved (left) [Unpaired Student's $t$-test. $\left.t_{16}=-0.670, \mathrm{NS}\right]$, mean velocity (middle) $\left[t_{16}=0.304, \mathrm{NS}\right]$ and $\%$ time spent in the center (right) $\left[t_{15}=-1.000, N S\right]$ in a novel openfield in WT $(n=9)$ and $\alpha 5$ SNP $(n=8-9)$ rats. c Percentage time spent in the light side (left) [Unpaired Student's $t$-test. $t_{18}=-0.093$, $\mathrm{NS}$ ], number of transitions (middle) $\left[t_{18}=0.391, N S\right.$ ] and latency to the first entry into the light side (right) $\left[t_{15}=-0.859, N S\right.$ in a dark-light box in WT $(n=12)$ and $\alpha 5 \operatorname{SNP}(n=8)$ rats. Data are mean + s.e.m

Rats were next submitted to several sessions of reinstatement of food seeking. a5SNPs had a significantly higher level of reinstatement than WTs in response to food priming, as observed on both AL presses (Fig. 5e, left) and relapse index (Fig. 5e, right).
Post hoc confirmed increased AL pressing in both groups after food priming and higher AL responding in a5SNP rats compared to WTs in relapse conditions. Re-exposure to the visual cue previously associated with food delivery also differentially affected $A L$ pressing in WT and a5SNP rats (Fig. $5 f$, left), with increased AL pressing observed only in a5SNPs. Cue-induced relapse index was also higher in a5SNPs compared to WTs (Fig. 5f, right). Nicotine was shown to regulate appetite and food intake [32]. To test whether nicotine could regulate food-seeking relapse and "rescue" the high-relapsing phenotype observed in a5SNPs, we exposed rats to nicotine (or saline) before submitting them to a last food priming-induced reinstatement of food seeking session (Fig. 5g). Again, we found that food priming significantly induced relapsing only in a5SNPs, as observed on both AL press number and relapse index. Nicotine decreased $A L$ pressing in response to food priming in both groups. The relapse index was also found significantly decreased by nicotine in both groups. These data reveal that the rs16969968 not only influences behaviors oriented towards drugs of abuse but is also associated with impairments in food-reward processing, including increased relapse to food seeking after extinction, which can be regulated by nicotine intake.

\section{DISCUSSION}

Evidence for a link between the a5SNP (rs16969968), a frequent coding variant at a highly conserved site in the $\mathrm{nAChR}$ second intracellular loop, and smoking risk is extremely robust. Here we show that this variant impacts responses to other reinforcers than nicotine, enhancing appetence for food and increasing alcohol addiction-like behaviors in rats. This polymorphism may have multiple phenotypic consequences contributing to several reward-related disorders and their comorbidity.

The a5SNP was previously shown to cause a partial loss of function of a5 containing $n A C h R s$ ( $a 5^{*} n A C h R s$ ) in response to nicotinic agonists [7, 22, 23, 33], including in human induced pluripotent stem cell (iPSC)-derived midbrain dopaminergic (DA) neurons [24], and was associated with consumption of increased amounts of high dose nicotine and increased relapse to nicotine seeking after extinction of nicotine SA in rodents [14,34]. Here, we demonstrate that a5SNP-induced addiction-like phenotypes, in particular increased relapse after extinction, are not specific to nicotine but are also observed for alcohol and food. Alcohol and tobacco use are highly correlated. Alcoholics are three times more likely to smoke than the general population [3], and at higher risk to die from smoking-related illnesses than from alcohol-related causes [35]. Several mechanisms have been proposed to underlie alcohol and tobacco addiction comorbidity, including cross-cue conditioning, cross-tolerance and -reinforcement [36, 37] and nicotinic modulation of alcohol effects [30, 38, 39]. Both alcohol and nicotine can modulate signalling pathways implicated in addiction through both distinct and common molecular targets. In fact, EtOH has been shown to act as an allosteric modulator of nAChRs and suggested to alter the balance between activation 
a

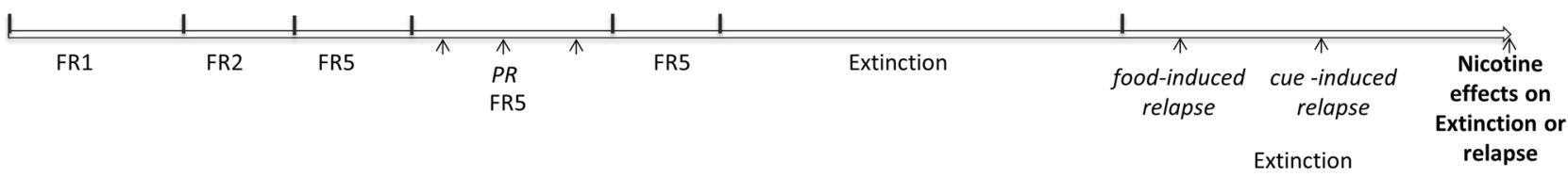

b

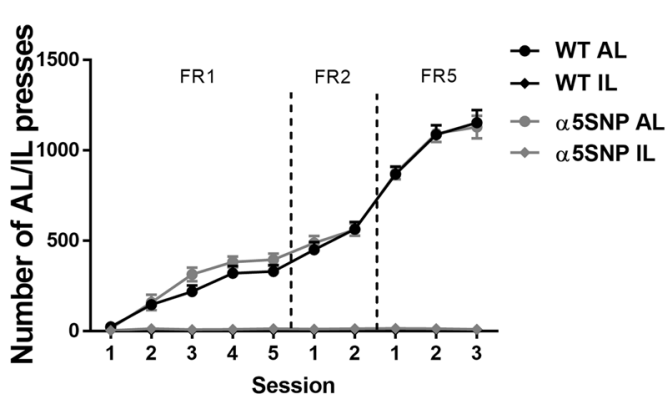

e

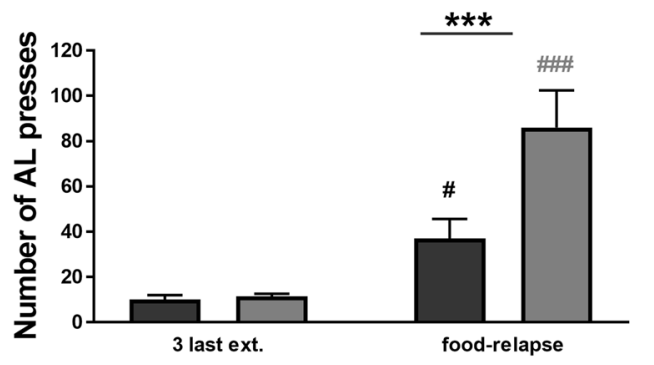

f

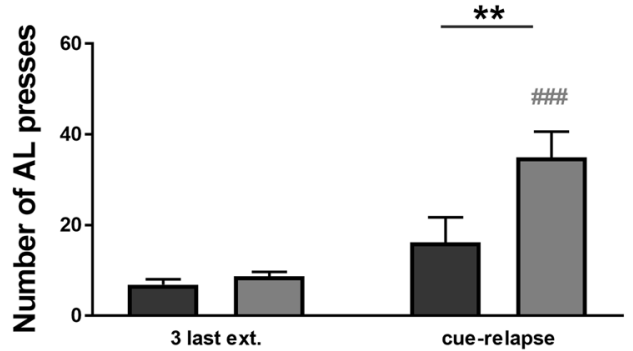

g

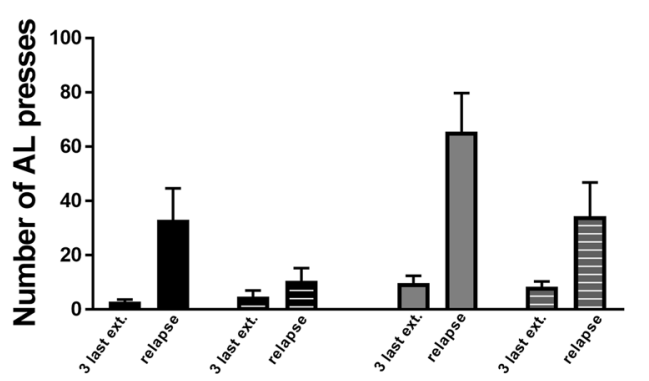

C

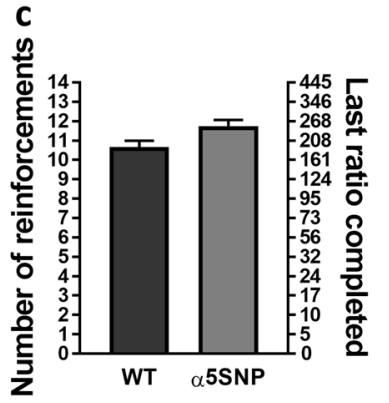

d

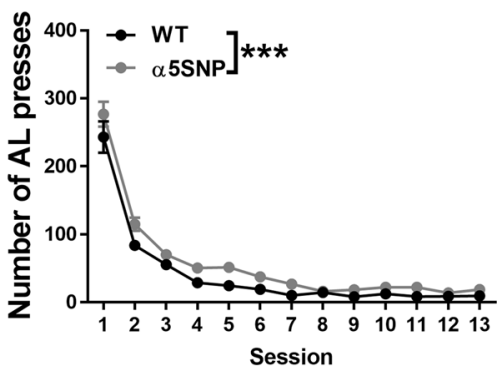

ש WT

口 $\alpha 5$ SNP
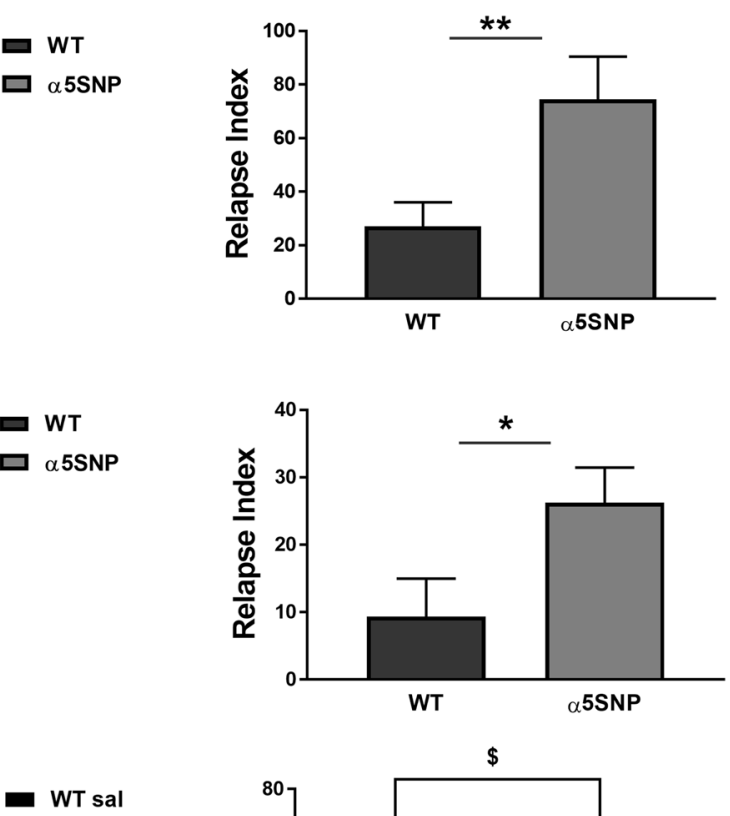

- WT nic

口 $\alpha 5$ SNP sal

口 $\alpha 5$ SNP nic

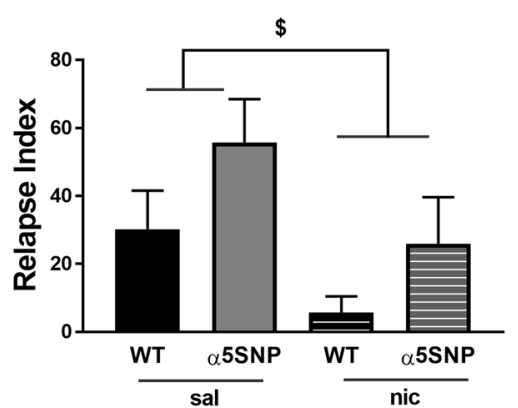

Fig. 5 Food operant self-administration. a Scheme of the procedure. b Number of active lever (AL) and inactive lever (IL) presses during acquisition in WT $(n=25)$ and $\alpha 5$ SNP $(n=18)$ rats [two-way repeated measure ANOVAs]. c Number of reinforcements and last ratio completed in WT $(n=24)$ and $\alpha 5 \mathrm{SNP}(n=18)$ rats under progressive ratio [Mann-Whitney. $z=2.312, p=0.068$ ]. $\mathbf{d}$ Number of AL presses during extinction in WT $(n=25)$ and $\alpha 5$ SNP $(n=18)$ rats [two-way repeated measure ANOVAs]. e Number of AL presses (left) [two-way repeated measure ANOVAs and Bonferroni post hoc WTs: ext. vs. rel., $p<0.05 ; \alpha 5 S N P s$ : ext. vs. rel., $p<0.0001$; Relapse: WT vs. $\alpha 5 S N P, p<0.001]$ and relapse index (right) [Unpaired Student's $t$-test. $t_{41}=2.849, p<0.01$ ] in WT $(n=25)$ and $\alpha 5 S N P(n=18)$ rats during food-induced food seeking reinstatement. $\mathbf{f}$ Number of AL presses (left) [two-way repeated measure ANOVAs and Bonferroni post hoc $\alpha 5$ SNPs: ext. vs. rel., $p<0.001$; Relapse: WT vs. $\alpha 5$ SNP, $p<0.01$ ] and relapse index (right) [Unpaired Student's $t$-test. $t_{41}=2.420, p<0.05$ ] in WT $(n=25)$ and $\alpha 5 S N P(n=18)$ rats during cue-induced food seeking reinstatement. $\mathbf{g}$ Number of AL presses (left) [three-way repeated-measure ANOVAs and Bonferroni post hoc. $\alpha 5$ SNPs: ext. vs. rel., $p<0.001$; Relapse: sal. vs. nic., $p<0.01$ ] and relapse index (right) [two-way repeated-measure ANOVAs] in salinetreated [WT $(n=13), \alpha 5 S N P(n=9)$ ] and nicotine-treated [WT $(n=12), \alpha 5 S N P(n=9)$ ] rats during food-induced food seeking reinstatement. Data are mean + s.e.m. Group effect (WT vs. $\alpha 5$ SNP): ${ }^{*} p<0.05,{ }^{* *} p<0.01,{ }^{* * *} p<0.001$. Session (ext. vs. rel.) effect: ${ }^{\text {s }} p<0.05$ in both groups, ${ }^{\#} p<0.05$ in WT rats (black), ${ }^{\# \#} p<0.001$ in $\alpha 5 S N P$ rats (gray) 
1914

and desensitization of nAChRs caused by nicotine [30]. Previous studies reported no alterations in EtOH intake in a5 knock-out (KO) mice in a drinking-in-the-dark paradigm [38, 40], although $\mathrm{EtOH}$ intake was actually decreased in these mice, in a similar paradigm, after a restraint stress [38]. The same study also reported decreased $\mathrm{EtOH}$-induced conditioned place preference in a5 KO mice. We previously found that a5 KO mice display increased anxiety-like behaviors [41], a phenotype we did not observe in a5SNP rats. Differences in stress and anxiety levels may contribute to differences in the response to $\mathrm{EtOH}$ reward. It would be of interest to further study a possible impact of the variant on the link between stress response and drug intake. Importantly, the partial loss of function of $a 5^{*} \mathrm{nAChRs}$ resulting from the a5SNP $[7,22,23,33]$ is likely to have different consequences compared to the complete absence of such receptors in a KO animal. Here, we show that a5SNP rats exhibit greater appetence and motivation for EtOH and increased relapse to EtOH seeking after extinction, the latter being associated with increased activation of the insula. The insula was proposed to integrate internal and external stimuli into interoceptive states to control motivated behavior, including drug craving [42-44]. It was previously implicated in alcohol interoceptive effect, SA and seeking behavior [43, 45, 46]. The a5SNP may potentiate alcohol addiction-like behaviors through direct alteration of alcohol-nAChR interaction, or indirectly through the modulation of acetylcholine control of neuronal activity, notably within the insular cortex. Interestingly, the anterior agranular insula has also been implicated in relapse to nicotine seeking [47]. Since the agranular insula receives multimodality sensory inputs, including from the primary olfactory cortex [48], sensitivity to EtOH may be altered in a5SNP rats through altered olfactory processing. It has also been established that the a5SNP decreases the sensitivity to nicotine aversive effects in humans [49], an effect further supported by preclinical studies $[14,34]$. It may be the case that aversive effects of alcohol are also diminished in a5SNP carriers, which may contribute to the higher EtOH drinking observed during the first two-bottle choice session in a5SNP rats and to their propensity for EtOH selfadministration, notably at high doses. Moreover, even though anxiety and locomotor activity in a novel environment were found unaltered in a5SNP rats in the present study, other behavioral traits previously associated with vulnerability to drug abuse, such as novelty preference $[31,50]$, may be altered in these rats and contribute to their initial preference for ethanol, which will need further investigation. It is well characterized that alcohol consumption is an important cause of relapse to smoking following smoking cessation [51, 52]. An impact of the a5SNP on alcohol drinking may thus also partly explain the strong influence of this polymorphism on smoking dependence, in addition to its direct consequences on nicotine effects. Further investigation for a possible association between the a5SNP and alcohol abuse, notably focusing on relapse rates and delays, may be of great importance.

Our present study further reveals an impact of the a5SNP on the response to natural reward, beyond its influence on nicotine and alcohol addiction-like behaviors. This suggests that the nAChR dysfunction associated with this variant alters acetylcholine modulation of reward pathways, affecting reinforcer processing in general. This polymorphism may also contribute to aberrant learning processes and to stronger associative memories that underlie reinforcer seeking [53]. Here, we demonstrate that the a5SNP is associated with resistance to previously acquired operant behavior extinction and increased food seeking relapse. Many smokers report that they consume tobacco to control appetite $[32,54]$. Weight control is cited as the primary reason to start smoking in teenage girls in the United States [55], and weight gain is perceived as a significant impediment for smoking cessation $[32,54,56,57]$. Nicotine also decreases food intake and body weight in mice [58]. Accordingly, we further show that nicotine reduces the intensity of food seeking relapse after extinction. To our knowledge, there is no study to date assessing a possible link between the a5SNP and eating behavior or disorders in humans. However, an increased BMI in non-smoking a5SNP carriers has been reported, while the BMI was decreased in carriers who are smokers [25]. Higher BMI increases the risk for tobacco dependence [59]. Our present data raise the hypothesis of an additional operating mode of the a5SNP for increased risk for heavy smoking, by contributing to a sub-population of comorbid eating and tobacco use disorders [60].

The present study demonstrates that the rs16969968 impacts alcohol addiction-related processes and appetence for food in rats, in addition to direct consequences on the brain's response to nicotine. These data call for new human genetics studies to refine our knowledge of the influence of this variant in psychiatric subpopulations including alcohol addicts and patients with eating disorders. Here we restricted our preclinical investigations on male subjects from one genetic background to limit the number of animals used. It would be important, in future human studies, to assess the effect of this variant according to gender and genetic ancestry. Finally, since this polymorphism decreases the response of $a 5^{*} \mathrm{nAChRs}$ to agonists, positive allosteric modulators (PAMs) of these receptors, by potentiating the effects of their primary ligands, may represent novel therapeutic strategy to address several psychiatric disorders. Such novel therapeutic approach could also lead to more favorable outcomes for comorbid issues in smokers.

\section{FUNDING AND DISCLOSURE}

This work was supported by the Institut Pasteur, Centre National de la Recherche Scientifique UMR 3571, Fondation pour la recherche en alcoologie, Fondation de la Recherche Médicale (SPF20140129365 and DPA20140629803), Agence Nationale de la Recherche (ANR), Neuroscience and BLANC, LABEX BIO-PSY, FP7 ERANET program NICO-GENE grant agreement convention ANR $\mathrm{n}^{\circ}$ 2010-NEUR-004-01, European Commission FP7 RTD Project HEALTH-2009-Neurocyp.08-202088 grant 242167, French National Cancer Institute grant CANCEROPOLE IDF 2016-1-TABAC-01-IP-1 MASKOS, ANR programme SNP-NIC, European ERANET programme iPS\&BRAIN (all to U.M.). The laboratory of U.M. is part of the Ecole des Neurosciences de Paris lle-de-France RTRA network. U.M. is a member of the LABEX BIO-PSY. This work was supported by French state funds managed by the ANR within the Investissements d'Avenir programme under reference ANR-11IDEX-0004-02. The authors declare no competing interests.

\section{ACKNOWLEDGEMENTS}

We thank Camille Ponthieu and Quentin Rodriguez for help with SA experiments, Romain Icick for helpful discussion, and the Institut de la Vision Platform for nanozoomer imaging.

\section{ADDITIONAL INFORMATION}

Supplementary Information accompanies this paper at (https://doi.org/10.1038/ s41386-019-0462-0).

Publisher's note: Springer Nature remains neutral with regard to jurisdictional claims in published maps and institutional affiliations.

\section{REFERENCES}

1. Yucel $M$, Oldenhof $E$, Ahmed SH, Belin D, Billieux J, Bowden-Jones $H$, et al. A transdiagnostic dimensional approach towards a neuropsychological assessment for addiction: an international Delphi consensus study. Addiction 2019;114:1095-1109. 
2. Ziedonis DM, Guydish J, Williams J, Steinberg M, Foulds J. Barriers and solutions to addressing tobacco dependence in addiction treatment programs. Alcohol Res Health. 2006;29:228-35.

3. Grant BF, Hasin DS, Chou SP, Stinson FS, Dawson DA. Nicotine dependence and psychiatric disorders in the United States: results from the national epidemiologic survey on alcohol and related conditions. Arch Gen Psychiatry. 2004;61:1107-15.

4. Van Skike CE, Maggio SE, Reynolds AR, Casey EM, Bardo MT, Dwoskin LP, et al. Critical needs in drug discovery for cessation of alcohol and nicotine polysubstance abuse. Prog Neuropsychopharmacol Biol Psychiatry. 2016;65:269-87.

5. Changeux JP. Nicotine addiction and nicotinic receptors: lessons from genetically modified mice. Nat Rev Neurosci. 2010;11:389-401.

6. Berrettini WH, Doyle GA. The CHRNA5-A3-B4 gene cluster in nicotine addiction. Mol Psychiatry. 2012;17:856-66.

7. Bierut $L$, Stitzel JA, Wang JC, Hinrichs AL, Grucza RA, Xuei X, et al. Variants in nicotinic receptors and risk for nicotine dependence. Am J Psychiatry. 2008;165:1163-71.

8. Stevens VL, Bierut LJ, Talbot JT, Wang JC, Sun J, Hinrichs AL, et al. Nicotinic receptor gene variants influence susceptibility to heavy smoking. Cancer Epidemiol Biomark Prev. 2008;17:3517-25.

9. Brunzell DH, Stafford AM, Dixon $\mathrm{Cl}$. Nicotinic receptor contributions to smoking: insights from human studies and animal models. Curr Addict Rep. 2015;2:33-46.

10. Weiss RB, Baker TB, Cannon DS, von Niederhausern A, Dunn DM, Matsunami N et al. A candidate gene approach identifies the CHRNA5-A3-B4 region as a risk factor for age-dependent nicotine addiction. PLoS Genet. 2008;4:e1000125.

11. Improgo MR, Scofield MD, Tapper AR, Gardner PD. The nicotinic acetylcholine receptor CHRNA5/A3/B4 gene cluster: dual role in nicotine addiction and lung cancer. Prog Neurobiol. 2010;92:212-26.

12. Saccone SF, Hinrichs AL, Saccone NL, Chase GA, Konvicka K, Madden PA, et al. Cholinergic nicotinic receptor genes implicated in a nicotine dependence association study targeting 348 candidate genes with 3713 SNPs. Hum Mol Genet. 2007;16:36-49.

13. Greenbaum L, Lerer B. Differential contribution of genetic variation in multiple brain nicotinic cholinergic receptors to nicotine dependence: recent progress and emerging open questions. Mol Psychiatry. 2009;14:912-45.

14. Forget B, Scholze P, Langa F, Morel C, Pons S, Mondoloni S, et al. A human polymorphism in CHRNA5 is linked to relapse to nicotine seeking in transgenic rats. Curr Biol. 2018;28:3244-53 e7.

15. Chen X, Chen J, Williamson VS, An SS, Hettema JM, Aggen SH, et al. Variants in nicotinic acetylcholine receptors alpha5 and alpha3 increase risks to nicotine dependence. Am J Med Genet B Neuropsychiatr Genet. 2009;150B:926-33.

16. Hallfors J, Loukola A, Pitkaniemi J, Broms U, Mannisto S, Salomaa V, et al. Scrutiny of the CHRNA5-CHRNA3-CHRNB4 smoking behavior locus reveals a novel association with alcohol use in a Finnish population based study. Int J Mol Epidemiol Genet. 2013:4:109-19.

17. Sherva R, Kranzler HR, Yu Y, Logue MW, Poling J, Arias AJ, et al. Variation in nicotinic acetylcholine receptor genes is associated with multiple substance dependence phenotypes. Neuropsychopharmacology. 2010;35:1921-31.

18. Wang JC, Grucza R, Cruchaga C, Hinrichs AL, Bertelsen S, Budde JP, et al. Genetic variation in the CHRNA5 gene affects mRNA levels and is associated with risk for alcohol dependence. Mol Psychiatry. 2009;14:501-10.

19. Bossert JM, Marchant NJ, Calu DJ, Shaham Y. The reinstatement model of drug relapse: recent neurobiological findings, emerging research topics, and translational research. Psychopharmacol (Berl). 2013;229:453-76.

20. de Kloet SF, Mansvelder HD, De Vries TJ. Cholinergic modulation of dopamine pathways through nicotinic acetylcholine receptors. Biochem Pharm. 2015;97:425-38.

21. Grasing K. A threshold model for opposing actions of acetylcholine on reward behavior: molecular mechanisms and implications for treatment of substance abuse disorders. Behav Brain Res. 2016;312:148-62.

22. Kuryatov A, Berrettini W, Lindstrom J. Acetylcholine receptor (AChR) alpha5 subunit variant associated with risk for nicotine dependence and lung cancer reduces (alpha4beta2)(2)alpha5 AChR function. Mol Pharm. 2011;79:119-25.

23. Frahm S, Slimak MA, Ferrarese L, Santos-Torres J, Antolin-Fontes B, Auer S, et al. Aversion to nicotine is regulated by the balanced activity of beta4 and alpha5 nicotinic receptor subunits in the medial habenula. Neuron. 2011;70:522-35.

24. Deflorio C, Blanchard S, Carisi MC, Bohl D, Maskos U. Human polymorphisms in nicotinic receptors: a functional analysis in iPS-derived dopaminergic neurons. FASEB J. 2017;31:828-39.

25. Taylor AE, Morris RW, Fluharty ME, Bjorngaard JH, Asvold BO, Gabrielsen ME, et al. Stratification by smoking status reveals an association of CHRNA5-A3-B4 genotype with body mass index in never smokers. Plos Genet. 2014;10:e1004799.

26. Hopf FW, Chang SJ, Sparta DR, Bowers MS, Bonci A. Motivation for alcohol becomes resistant to quinine adulteration after 3 to 4 months of intermittent alcohol self-administration. Alcohol Clin Exp Res. 2010;34:1565-73.
27. Carnicella S, Ron D, Barak S. Intermittent ethanol access schedule in rats as a preclinical model of alcohol abuse. Alcohol. 2014;48:243-52.

28. Kovacs KJ. Measurement of immediate-early gene activation-c-fos and beyond. J Neuroendocrinol. 2008;20:665-72.

29. Doyon WM, Thomas AM, Ostroumov A, Dong Y, Dani JA. Potential substrates for nicotine and alcohol interactions: a focus on the mesocorticolimbic dopamine system. Biochem Pharm. 2013;86:1181-93.

30. Klenowski PM, Tapper AR. Molecular, neuronal, and behavioral effects of ethanol and nicotine interactions. Handb Exp Pharmacol. 2018. https://doi.org/10.1007/ 164_2017_89

31. Belin D, Belin-Rauscent A, Everitt BJ, Dalley JW. In search of predictive endophenotypes in addiction: insights from preclinical research. Genes Brain Behav. 2016;15:74-88.

32. Picciotto MR, Mineur YS. Nicotine, food intake, and activation of POMC neurons. Neuropsychopharmacology. 2013;38:245-45.

33. Sciaccaluga M, Moriconi C, Martinello K, Catalano M, Bermudez I, Stitzel JA, et al. Crucial role of nicotinic alpha5 subunit variants for $\mathrm{Ca} 2+$ fluxes in ventral midbrain neurons. FASEB J. 2015;29:3389-98.

34. Morel C, Fattore L, Pons S, Hay YA, Marti F, Lambolez B, et al. Nicotine consumption is regulated by a human polymorphism in dopamine neurons. Mol Psychiatry. 2014;19:930-6.

35. Hurt RD, Offord KP, Croghan IT, Gomez-Dahl L, Kottke TE, Morse RM, et al. Mortality following inpatient addictions treatment. Role of tobacco use in a community-based cohort. JAMA. 1996;275:1097-103.

36. Adams S. Psychopharmacology of tobacco and alcohol comorbidity: a review of current evidence. Curr Addict Rep. 2017:4:25-34.

37. Drobes DJ. Cue reactivity in alcohol and tobacco dependence. Alcohol Clin Exp Res. 2002;26:1928-9.

38. Dawson A, Wolstenholme JT, Roni MA, Campbell VC, Jackson A, Slater C, et al Knockout of alpha 5 nicotinic acetylcholine receptors subunit alters ethanolmediated behavioral effects and reward in mice. Neuropharmacology. 2018;138:341-48.

39. Tolu S, Marti F, Morel C, Perrier C, Torquet N, Pons S, et al. Nicotine enhances alcohol intake and dopaminergic responses through beta2* and beta $4 *$ nicotinic acetylcholine receptors. Sci Rep. 2017;7:45116.

40. Santos N, Chatterjee S, Henry A, Holgate J, Bartlett SE. The alpha5 neuronal nicotinic acetylcholine receptor subunit plays an important role in the sedative effects of ethanol but does not modulate consumption in mice. Alcohol Clin Exp Res. 2013;37:655-62.

41. Besson M, Guiducci S, Granon S, Guilloux JP, Guiard B, Reperant C, et al. Alterations inalpha5* nicotinic acetylcholine receptors result in midbrain- and hippocampus-dependent behavioural and neural impairments. Psychopharmacol (Berl). 2016;233:3297-314.

42. Craig AD. How do you feel-now? The anterior insula and human awareness. Nat Rev Neurosci. 2009;10:59-70.

43. Paulus MP, Stewart JL. Interoception and drug addiction. Neuropharmacology. 2014;76 Pt B:342-50.

44. Belin-Rauscent A, Daniel ML, Puaud M, Jupp B, Sawiak S, Howett D, et al. From impulses to maladaptive actions: the insula is a neurobiological gate for the development of compulsive behavior. Mol Psychiatry. 2016;21:491-9.

45. Droutman V, Read SJ, Bechara A. Revisiting the role of the insula in addiction. Trends Cogn Sci. 2015;19:414-20.

46. Jaramillo AA, Van Voorhies K, Randall PA, Besheer J. Silencing the insular-striatal circuit decreases alcohol self-administration and increases sensitivity to alcohol. Behav Brain Res. 2018:348:74-81.

47. Pushparaj A, Kim AS, Musiol M, Trigo JM, Le Foll B. Involvement of the rostral agranular insular cortex in nicotine self-administration in rats. Behav Brain Res. 2015;290:77-83.

48. Mori K, Manabe H, Narikiyo K, Onisawa N. Olfactory consciousness and gamma oscillation couplings across the olfactory bulb, olfactory cortex, and orbitofrontal cortex. Front Psychol. 2013;4:743.

49. Jensen KP, DeVito EE, Herman Al, Valentine GW, Gelernter J, Sofuoglu M. A CHRNA5 smoking risk variant decreases the aversive effects of nicotine in humans. Neuropsychopharmacology. 2015;40:2813-21.

50. Perez-Martinez IO, Acevedo-Roque CR, Montes-Angeles CD, Martinez M, Miranda F. Mental nerve injury induces novelty seeking behaviour leading to increasing ethanol intake in Wistar rats. Arch Oral Biol. 2019;99:66-72.

51. Businelle MS, Ma P, Kendzor DE, Frank SG, Wetter DW, Vidrine DJ. Using Intensive longitudinal data collected via mobile phone to detect imminent lapse in smokers undergoing a scheduled quit attempt. J Med Internet Res. 2016;18:e275.

52. Lynch KL, Twesten JE, Stern A, Augustson EM. Level of alcohol consumption and successful smoking cessation. Nicotine Tob Res. 2018. https://doi.org/10.1093/ ntr/nty 142

53. Milton AL, Everitt BJ. The persistence of maladaptive memory: addiction, drug memories and anti-relapse treatments. Neurosci Biobehav Rev. 2012:36:1119-39. 
Profound alteration in reward processing due to a human polymorphism in...

$\mathrm{M}$ Besson et al.

1916

54. Zoli M, Picciotto MR. Nicotinic regulation of energy homeostasis. Nicotine Tob Res. 2012;14:1270-90.

55. Fulkerson JA, French SA. Cigarette smoking for weight loss or control among adolescents: gender and racial/ethnic differences. J Adolesc Health. 2003;32:306-13.

56. Clark MM, Decker PA, Offord KP, Patten CA, Vickers KS, Croghan IT, et al. Weight concerns among male smokers. Addict Behav. 2004;29:1637-41.

57. Pomerleau CS, Zucker AN, Stewart AJ. Characterizing concerns about postcessation weight gain: results from a national survey of women smokers. Nicotine Tob Res. 2001;3:51-60.
58. Mineur YS, Abizaid A, Rao Y, Salas R, DiLeone RJ, Gundisch D, et al. Nicotine decreases food intake through activation of POMC neurons. Science. 2011;332:1330-2.

59. Taylor AE, Richmond RC, Palviainen $T$, Loukola A, Wootton R, Kaprio J et al. The effect of body mass index on smoking behaviour and nicotine metabolism: a Mendelian randomization study. Hum Mol Genet. 2018. https://doi.org/10.1093/ hmg/ddy434

60. Solmi M, Veronese N, Sergi G, Luchini C, Favaro A, Santonastaso P, et al. The association between smoking prevalence and eating disorders: a systematic review and meta-analysis. Addiction. 2016;111:1914-22. 